\title{
Netnografia no Programa de Inclusão Digital AcessaSP
}

Brasilina Passarelli

Professora titular e chefe do Departamento de Biblioteconomia e Documentação da Escola de Comunicações e Artes da Universidade de São Paulo. Coordenadora Científica do Núcleo de Pesquisa de Novas Tecnologias da Comunicação Aplicadas à Educação (NAP) "Escola do Futuro"/USP.

E-mail: lina@futuro.usp.br

Antonio Helio Junqueira

Doutorando em Ciências da Comunicação pela Escola de Comunicação e Artes da Universidade de São Paulo. Mestre em Comunicação e Práticas de Consumo pela Escola Superior de Propaganda e Marketing (ESPM). Consultor de Empresas da Junqueira e Peetz Consultores Ltda.

E-mail: heliojunqueira@usp.br

Rodrigo Eduardo Botelho Francisco

Doutorando em Ciências da Comunicação pela Escola de Comunicação e Artes da Universidade de São Paulo (ECA/USP). Diretor de Comunicação Social da Universidade Federal de São Carlos (UFSCAR).

E-mail: rodrigobotelho@usp.br

Resumo: Na atualidade, as Tecnologias de Informação e Comunicação (TIC) configuram-se como um importante território de produção e transformação sociocultural, no qual se engendram novos valores, sentidos e práticas sociais pautados pela colaboração e interação em rede. Em um contexto de discussão sobre indicadores de acesso ao computador e à Internet, a presente pesquisa vale-se da netnografia para abordar as literacias informacionais emergentes e a inclusão digital de idosos no Programa AcessaSP.

Palavras-chave: Cibercultura, inclusão digital, netnografia, literacias emergentes na Web 2.0, idosos e as TIC.
Resumo: Nowadays ICT become an important territory for the production and socio-cultural transformation, with the production of new values, meanings and social practices guided by collaboration and network interaction. In a context of discussion about indicators of computer access and the Internet, this research uses netnography to map the emerging informational literacies and digital inclusion of older people in the digital inclusion program AcessaSP.

Keywords: Ciberculture, digital inclusion, netnography, Web 2.0 emerging literacies, elderly people digital literacies.

\section{A INCLUSÃO DIGITAL NA TERCEIRA IDADE}

A sociedade em rede, que caracteriza a contemporaneidade, se constitui e 
1. CASTELLS, Manuel. A sociedade em rede. 2. ed. São Paulo: Paz e Terra, 1999. KERKHOVE, Derrick de. A pele da cultura: uma investigação sobre a nova realidade electrónica. Lisboa: Relógio D'água, 1997.

2. PASSARELLI, Brasilina. Literacias emergentes nas redes sociais: estado da arte e pesquisa qualitativa no observatório da cultura digital. In: PASSARELLI, Brasilina; AZEVEDO, José (org.). Atores em rede olhares luso-brasileiros. São Paulo: Editora Senac 2010; PASSARELLI, Brasilina. School of the Future. Research Laboratory/USP: action research and emerging literacies studies in WEB 2.0 environments [Escola do Futuro - Laboratório de Pesquisa/USP. pesquisa-ação e estudos de literacia digital na Web 2.0]. The Journal of Community Informatics v. 7, ns. 1 \& 2, 2010/2011. Disponível em: <http:// ci-journal.net/index.php/ ciej/article/view/646>. Acesso em: 14 out. 2011. PASSARELLI, Brasilina SALLA, Thiago Mio; TAVERNARI, Mariana. Literacias emergentes dos atores em rede: etnografia virtual com idosos no Programa de Inclusão Digital AcessaSP. Revista Prisma.com, n. 13, 2010. Disponível em: <http:// revistas.ua.pt/index. $\mathrm{php} / \mathrm{prismacom/article/}$ view/789>. Acesso em: 10 maio 2011.

3. COMITÊ GESTOR DA INTERNET NO BRASIL (CGI.br). TIC Domicílios e Empresas 2010: pesquisa sobre o uso de tecnologias de informação e comunicação no Brasil. Disponível em: $<$ http://cetic.br/usuarios/ tic/2010-total-brasil/index.htm>. Acesso em: 13 jul. 2011.
Informação e Comunicação (TIC), assim como pelo aumento exponencial das conexões digitais entre os indivíduos e as diferentes comunidades a que pertencem, reconfigurando a estrutura social, suas relações de poder e a própria cultura $^{1}$. Ao mesmo tempo, contudo, as questões da exclusão social permanecem relevantes e urgentes na agenda política do desenvolvimento socioeconômico e cultural em todo o mundo.

A exclusão digital - que abrange amplas parcelas de populações de países economicamente marginalizados, mas também estratos e comunidades específicas em todas as sociedades - tem merecido múltiplos olhares no âmbito da pesquisa acadêmica atual. Entre eles, as abordagens acerca da aquisição e do desenvolvimento das literacias emergentes na Web 2.0 têm constituído importante questão de pesquisa do Núcleo das Tecnologias de Comunicação e Informação Aplicadas à Educação "Escola do Futuro USP" (NAP EF/USP), na medida em que sinalizam para as possibilidades de engajamento do indivíduo em suas comunidades, seus contextos, valores, demandas e interações políticas e sociais².

Segundo dados da Organização das Nações Unidas (ONU), publicados em março de 2011, o acesso global à Internet em 2010 abrangeu mais de dois bilhões de usuários, correspondendo a praticamente um terço da população mundial. Tal contingente populacional representou, ainda, o dobro do número de usuários registrados em 2005. O relatório da ONU, no entanto, também revela que apenas $21 \%$ da população dos países em desenvolvimento está conectada on-line, contra $71 \%$ da população dos países desenvolvidos - e que a penetração da Internet entre usuários na África não atingiu mais do que 9,6\% da população do continente, número aquém da média mundial (30\%) e da média dos países em desenvolvimento (21\%).

No Brasil, segundo pesquisa de 2010 do Comitê Gestor da Internet ${ }^{3}$ (CGI. br), $35 \%$ dos domicílios possuem computador, sendo esse percentual elevado para $39 \%$ na área urbana. No mesmo ano, uma parcela de $27 \%$ dos lares declarou-se também conectada à Internet, porcentagem esta que ascendeu, na área urbana, a 31\%. Assim, contabilizou-se que 4,5 milhões de domicílios brasileiros possuem computadores sem acesso à Internet. Há que se ressaltar, contudo, que o CGI.br estima uma expansão mínima de $52 \%$ nas conexões domésticas à Internet no Brasil até 2015. Em 2010, o número de internautas brasileiros, considerando também aqueles que acessam a rede a partir de locais públicos, ultrapassou 73 milhões de pessoas.

A população idosa brasileira é crescente e, culturalmente, tende a ser marginalizada na vida social - tanto no contexto familiar quanto profissional e comunitário. A inclusão digital dos indivíduos com idade igual ou superior a 60 anos de idade sinaliza para a reintegração mais dinâmica e participativa dessa população no corpo social, desde que superadas as dificuldades não apenas de acesso às TIC, mas também aquelas relativas à sua efetiva apropriação e uso na construção de sentidos e narrativas pessoais dos grupos de identificação e pertencimento. Reporta-se, portanto, ao conceito das literacias digitais e sua aplicabilidade no estudo da educação, emancipação e integração social dos sujeitos na contemporaneidade. 
Para Gislter ${ }^{4}$, a literacia digital não se esgota na aquisição de habilidades, mas expande-se para as formas como estas são incorporadas e efetivamente utilizadas na vida cotidiana dos indivíduos.

Estudos realizados ao longo das duas últimas décadas vêm corroborando a ideia de que o desenvolvimento das literacias digitais exige dos indivíduos não apenas habilidades motoras e de interação pessoal, mas, também, cognitivas - como aquelas relacionadas ao raciocínio, intelecto, capacidade de análise, avaliação e crítica $^{5}$. Dessa forma, as literacias digitais devem ser entendidas em sua multiplicidade.

A compreensão das novas literacias digitais exige foco na prática social e na aplicação da crítica, da reflexão, do julgamento e da cognição - muito distantes, portanto, daquilo que pode ser explicado apenas pelas habilidades inatas e cognitivas ${ }^{6}$. Neste contexto, a aquisição das literacias ligadas ao campo informacional reporta-se, essencialmente, à conquista de habilidades e competências para a construção de sentidos, viabilizando o aprendizado e o raciocínio independentes e autônomos ${ }^{7}$.

Outros autores contemporâneos também têm defendido que o conceito de literacia aplicado ao campo informacional não deve ser definido com base em habilidades e, ainda menos, em um conjunto de habilidades descontextualizadas, aleatoriamente adquiridas e acumuladas pelos indivíduos. Pelo contrário, deve ser pensado enquanto processo holístico, experienciado pelo sujeito nas suas interações com a tecnologia, de maneira autoconsciente ${ }^{8}$ e mediado simultaneamente pelas relações sociais, físicas e textuais com a informação ${ }^{9}$. A este indivíduo em permanente aprendizado informacional ao longo de toda a sua existência se associam, ainda, aspectos éticos e de responsabilidade social na obtenção, apropriação, uso, transformação, armazenamento e disseminação da informação ${ }^{10}$.

Nesse sentido, novos conceitos sobre as literacias digitais sinalizam para o engajamento dos indivíduos com suas comunidades, seus contextos, valores, demandas e interações políticas e sociais ${ }^{11}$. A aquisição e, mais do que isso, o desenvolvimento permanente das literacias informacionais, sob essa ótica, revestem-se de uma perspectiva emancipadora e libertadora, na medida em que os agentes podem tornar-se sujeitos ativos de sua educação, aquisição de conhecimentos e atuação social ${ }^{12}$.

\section{O PROGRAMA DE INCLUSÃO DIGITAL ACESSASP}

Um dos principais projetos de pesquisa-ação conduzidos pelo NAP EF-USP ao longo dos últimos dez anos é o AcessaSP (http://www.acessasaopaulo.sp.gov. br) - programa financiado pelo governo do Estado de São Paulo através da Companhia de Processamento de Dados do Estado de São Paulo (Prodesp), focado prioritariamente na inclusão digital e no protagonismo social a partir da disponibilização de equipamentos, infraestrutura e acesso à Internet gratuita - contribuindo para o desenvolvimento social, cultural, intelectual e econômico
4. GILSTER, Paul. Digital literacy [Literacia digital]. San Francisco, CA: John Willey \& Sons, 1997.

5. GARDNER, Howard. A nova ciência da mente: uma história da revolução cognitiva. São Paulo: Editora da Universidade de São Paulo, 2003.

6. WARSCHAUER, Mark. Technology and social inclusion: rethinking the digital divide [Tecnologia e inclusão social: repensando a exclusão digital]. Massachusetts: MIT Press, 2003. MARTÍN-BARBERO, Jesús. Cartografias culturales de la sensibilidad y la tecnicidad [Cartografias culturais da sensibilidade e da tecnicidade]. Apostila do curso ministrado no Programa de Pós-Graduação em Ciências da Comunicação (ECA), Universidade de São Paulo, 2008.

7. SINGH, Jagtar. Sense-making: information literacy for lifelong learning and information management [Sensemaking: literacias informacionais para o gerenciamento da informação e aprendizado ao longo da vida]. Journal of Library and Information Technology, v. 28, n. 2, p. 13-17, mar. 2008. 8. WEBBER, Sheila; JOHNSTON, Bill. Conceptions of information literacy: new perspectives and implications [Conceitos de literacia informacional: novas perspectivas e implicações]. Journal of Information Science, v. 26, n. 6, p. 81-397, 2000.

9. STREET, Brian. Social literacies: critical approaches to literacy in development, ethnography and educación [Literacias Sociais: aproximações críticas das literacias em desenvolvimento, etnografia e educação]. London: Longman, 1995. LLOYD, Annemaree. Information literacy landscapes: an emerging picture 
[Panorama das Literacias Informacionais: um quadro emergente]. Journa of Documentation, v. 62, n. 5, p. 570-583, 2006

10. HARRIS, Benjamin Communities as necessity in information literacy development: challenging the standards [Comunidades como necessidade do desenvolvimento de literacias informacionais: desafiando os padrões]. The Journal of Academic Librarianship, v. 34, n. 3 , p. $248-255,2008$

11. HARTLEY, John. Communication, Cultural and Media Studies: the key concepts [Comunicação, Estudos Culturais e de Mídia: conceitos-chave]. Londres: Routledge, 2002

12. Dados disponíveis em: $<$ http://www.acessasaopaulo.sp.gov.br>. Acesso em: 16 out. 2011 dos cidadãos paulistas. De dimensões gigantescas, o AcessaSP acumula indicadores tais como: 58,34 milhões de atendimentos em dez anos de existência; 2,28 milhões de agentes cadastrados; 629 postos de atendimento (Infocentros e/ou Telecentros) em funcionamento e 52 em implantação; 543 municípios paulistas atendidos e 1.172 monitores capacitados ${ }^{12}$.

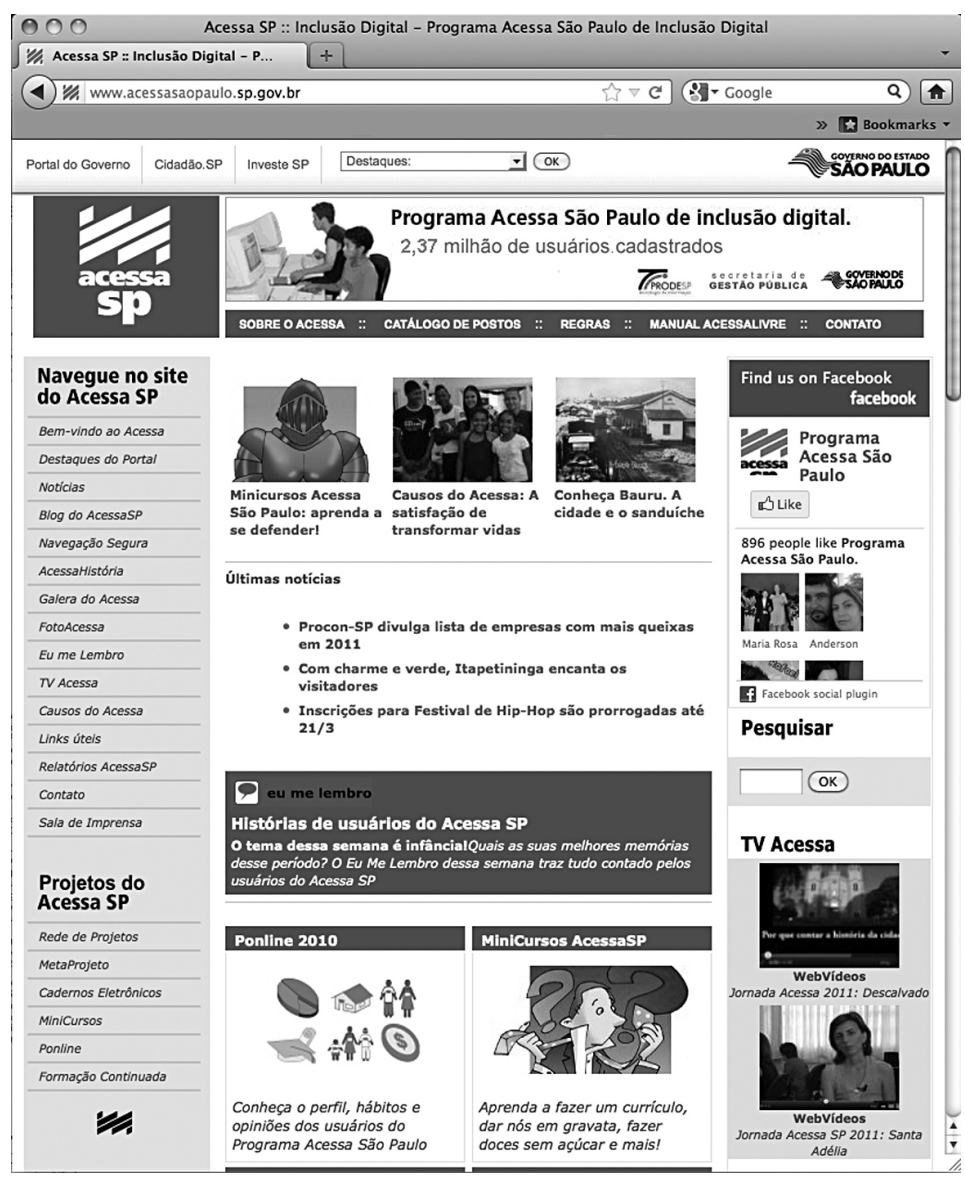

Homepage do site do Programa de inclusão digital AcessaSP.

Desde 2002, o AcessaSP realiza extensas pesquisas on-line intituladas PONLINE, sobre os usuários do programa, visando à identificação do seu perfil, seus usos e hábitos na Internet. Os resultados dessas pesquisas, disponíveis na rede, permitem análises da evolução do programa e de seus participantes. A PONLINE se utiliza, como ferramenta de pesquisa, de um questionário on-line composto de questões de múltipla escolha, de avaliação em escala e de perguntas abertas, aplicado em uma amostra aleatória simples dos usuários cadastrados ao longo de uma semana de funcionamento.

A partir da PONLINE é possível obter um conjunto de informações sobre como se tem dado a inclusão digital no AcessaSP e sobre como os participantes deste programa têm interagido com as TIC. Nesse sentido, a pesquisa dedicou-se a avaliar os dados disponíveis dos frequentadores com idade igual ou superior a 60 anos de idade - mostrando participação estável (de 1\% do 
total) no programa no período de 2006 a 2008, mas tendo dobrado a partir daí. Os indicadores do envelhecimento da população brasileira e o comprovado interesse crescente da população de terceira idade pelo uso dos recursos disponibilizados pela web justificam a presente pesquisa.

\section{A OPÇÃO PELA ABORDAGEM NETNOGRÁFICA}

A pesquisa sobre a cibercultura tem-se valido de metodologias apropriadas e adaptadas da Antropologia, que se legitimam pelo reconhecimento das novas socialidades promovidas em rede na contemporaneidade a partir dos usos e apropriações das TIC. Entre essas, é possível distinguir uma vertente mais orientada para a pesquisa nos campos da Antropologia, da Sociologia e da Comunicação, à qual se tem preferencialmente reservado a denominação de "etnografia virtual"13, e outra mais utilizada nos estudos do Consumo, do Marketing e da Administração, usualmente denominada "netnografia"14. O neologismo, originado da somatória das palavras inglesas "net" e "ethnography, foi cunhado por Bishop, Star, Neumann, Ignácio, Sandusky e Schatz, nos EUA, em $1995^{15}$, a partir da preocupação em descrever o comportamento de consumo de comunidades atuantes na Internet. Mais recentemente, a organização metodológica proposta por Robert Kozinets ${ }^{16}$ passou a constituir um dos principais aportes para os estudos não apenas dos hábitos de consumo virtuais, mas de toda a pesquisa netnográfica. No conjunto de procedimentos metodológicos estudados e recomendados por esse autor, destacam-se quatro diferentes etapas, descritas a seguir.

Inicialmente, inclui-se a entrée cultural - atividade que envolve a definição precisa do problema de pesquisa e do levantamento da comunidade a ser estudada - e a coleta e análise de dados, através das quais se busca compreender os sujeitos representados nas suas interações com o contexto cultural da comunidade on-line pesquisada. Os outros dois procedimentos metodológicos da netnografia, conforme proposta por Kozinets ${ }^{17}$, referem-se à ética na pesquisa, que inclui as apresentações do pesquisador ao grupo, a explicitação de seus objetivos e a obtenção de autorização do uso e divulgação dos dados em pesquisa. Finalmente, segue-se a etapa de validação dos resultados obtidos pelo investigador junto à comunidade pesquisada. Esta última tem o propósito de verificar a veracidade e pertinência do trabalho, conferindo-lhe confiabilidade e credibilidade. Se necessário, pode-se sugerir eventuais correções dos dados obtidos ou de sua análise e interpretação.

A presente pesquisa, realizada junto aos idosos frequentadores do Programa AcessaSP entre 2008 e 2009, concentra-se nas etapas iniciais do método netnográfico proposto por Kozinets: entrée cultural e coleta e análise de dados, com o objetivo de obter informações, conhecimento e compreensão a respeito da inserção desse público na cultura digital. Tem como propósito, também, avaliar a importância, oportunidade e pertinência de seu futuro aprofundamento em uma pesquisa mais abrangente no campo virtual, pela qual os pesquisadores poderão interagir de forma participativa com o público investigado.
13. HINE, Christine. Virtual Ethnography [Etnografia virtual]. London: Sage, 2000. HINE, Christine. Virtual Methods: Issues in Social Research on the Internet [Métodos virtuais: estudos em pesquisa social na Internet]. Oxford: Berg, 2005.

14. ROCHA, Paula Jung; MONTARDO, Sandra Portella. Netnografia: incursões metodológicas na cibercultura. e-COMPÓs: Revista da Associação Nacional dos Programas de Pós-Graduação em Comunicação, p. 13-22, dez. 2005. Disponível em: <http://www.compos.com.br/e-compos>. Acesso em: 22.11.2007.

15. BRAGA, Adriana. Usos e consumos de meios digitais entre participantes de weblogs: uma proposta metodológica. In: Anais do XVI Encontro da Compós, Curitiba, 2007. Disponível em: <http://compos.org.br/ data/biblioteca_162.pdf>. Acesso em: 06 mar. 2011.

16. KOZINETS, Robert. The Field behind the Screen: Using Netnography for Marketing Research in Online Communities OO campo atrás da tela: usando netnografia para pesquisas de Marketing em comunidades on-line]. Journal of Marketing Research, 39, February, p. 61-72, 2002. Disponível em: <http://www. nyu.edu/classes $/ \mathrm{bkg} / \mathrm{me}$ thods/netnography.pdf>. Acesso em: 15 out. 2011.

17. Ibid. 
comunicação \& educação • Ano XVII • número 1 • jan/jun 2012

A entrée cultural constitui-se em uma etapa metodológica prévia e delimitada pelo próprio pesquisador, necessária à sua preparação para o trabalho de campo. Nela, levantam-se os tópicos e as questões que se deseja analisar, bem como os tipos de comunidades e de participantes que podem prover respostas satisfatórias às questões de pesquisa.

Já a coleta e a análise de dados - que devem ocorrer concomitantemente à etapa de planejamento e preparo - podem incluir diferentes tipos e práticas. Para Kozinets ${ }^{18}$, as coletas de informações podem se referir a dados de arquivo (registros sobre a comunidade obtidos sem a influência direta do pesquisador, posteriormente filtrados quanto a sua relevância para o contorno da pesquisa); dados criados pela parceria entre pesquisador e comunidade (e que reflitam as interações e simbologias entre os membros da comunidade e entre esses e o próprio pesquisador); e dados de campo obtidos a partir dos relatos de experiências do próprio pesquisador. Quanto à análise, “... são cruciais a codificação e categorização dos dados levantados, comparação em busca de recorrências, contradições e desvios" ${ }^{\prime 19}$.

Para Kozinets ${ }^{20}$, a etnografia apresenta-se vantajosa em comparação a outros métodos de pesquisa qualitativa, por ser considerada flexível, não obstrutiva, rápida e menos exigente em termos de investimento de recursos humanos e financeiros.

\section{RESULTADOS E DISCUSSÃO}

Nos anos analisados, a população de idosos frequentadores do AcessaSP evidenciou acréscimo nos índices de presença do computador em suas residências. De fato, o percentual de idosos que declarou não possuir computador em casa decresceu de $59 \%$ do total dos usuários da faixa etária, em 2008, para 56\% em 2009. Entre esses mesmos anos de comparação, a presença de um único computador na residência também se elevou de $34 \%$ para $39 \%$. Em parte devido à maior penetração residencial dos computadores, o percentual de idosos que acessavam a internet a partir de suas casas elevou-se de $18 \%$, em 2008, para 23\%, em 2009.

A pesquisa revelou que os idosos passaram a usar mais significativamente a Internet como principal fonte de informação no período analisado. O percentual dos que assim o declararam saltou de 66\%, em 2008, para 74\%, em 2009.

18. Ibid.

19. VASCONCELLOS Marcelo Simão de; ARAÚJO, Inesita Soares de. Usos da etnografia em mundos virtuais baseados em imagem. RECIIS - Revista Eletrônica de Comunicação, Informação e Inovação em Saúde Rio de Janeiro, v. 5, n. 2, p. $75-85,2011$.

20. KOZINETS, op. cit.
De maneira mais específica, o crescimento do interesse e da receptividade aos conteúdos disponíveis na Internet foi observado em relação a questões relativas aos cuidados com a saúde (de 61\%, em 2008, para 67\%, em 2009), treinamento e educação (de 10\%, em 2008, para 16\%, em 2009) e serviços financeiros (de 41\%, em 2008, para 48\%, em 2009).

No tocante ao interesse e uso dos serviços governamentais on-line, observou-se expressivo crescimento de alguns serviços em face do decréscimo de outros, não indicando um comportamento padronizado. Entre os serviços ascendentes, observaram-se: a) consulta a informações sobre o programa Nota Fiscal 
Paulista, da Receita Estadual de São Paulo (40\%, em 2009); b) emissão de atestado de antecedentes criminais (de 14\%, em 2008, para 21\%, em 2009); c) de Boletins de Ocorrência (de 11\% para 13\%); d) de consultas sobre IPTU e outros impostos municipais (de 14\%, em 2008, para 15\%, em 2009). Por outro lado, mostraram-se decrescentes: a) consulta ao CPF (de $40 \%$ para 30\%) e b) declaração do Imposto de Renda (de 52\% para 42\%).

A pesquisa sobre o aprendizado do uso da internet pelos frequentadores do AcessaSP revelou que, entre 2008 e 2009, subiram os percentuais daqueles que o fizeram por conta própria (de $19 \%$ para $27 \%$ ) e dos que o fizeram com o auxílio de parentes (de 3\% para 13\%). No que tange à participação dos monitores em tal aprendizado, os percentuais decaíram de 50\% (em 2008) para $34 \%$ (em 2009). No mesmo período, observou-se aumento no percentual de frequentadores de cursos específicos para a utilização da Internet (de 18\%, em 2008, para 30\%, em 2009). Nota-se, portanto, crescimento da autonomia pessoal no uso dos recursos e potencialidades da Web 2.0.

As atividades de comunicação mais frequentemente utilizadas pelos idosos do AcessaSP no período analisado foram: a) enviar e receber e-mails (de $76 \%$ para 90\%); b) conversar via mensagens instantâneas (de 20\% para 30\%); c) conversar via recados/"scraps" (de 10\% para 15\%); d) participar de salas de bate-papo/chats (de 6\% para 15\%); e) criar ou atualizar blogs ou sites (de $8 \%$ para 15\%); f) participar de listas de discussão ou fóruns (de 6\% para $8 \%$ ); g) conversar usando programas de mensagem de voz (de $3 \%$ para $11 \%$ ). Observou-se, em 2009, o surgimento, com 7\% de participação nas respostas, da atividade "postar em microblog, tipo Twitter", que não aparecia em 2008. O percentual dos que não realizavam nenhuma atividade deste grupo decaiu de $18 \%$, em 2008, para 8\%, em 2009.

Vale observar que, em 2008, o percentual de frequentadores de faixa mais idosa do AcessaSP, e que não possuía nenhum perfil em redes sociais, era de $73 \%$ - tendo esse número decrescido para $43 \%$ no ano seguinte, refletindo principalmente a maior penetração do Orkut (de 21\% para 29\%). Quanto ao uso do YouTube, o percentual de idosos ascendeu de $2 \%$ para $9 \%$.

Observou-se, ainda, que a participação dos frequentadores da terceira idade na vida comunitária foi impactada positivamente pela apropriação e uso dos recursos da Internet. As afirmações de participação comunitária no período analisado cresceram de $48 \%$ para $63 \%$.

\section{CONSIDERAÇÕES FINAIS}

No Brasil, como na maioria dos países, pesquisas têm comprovado o crescente interesse, acesso e uso dos computadores e ferramentas de Internet pela população da terceira idade. Tais fenômenos têm-se feito acompanhar de maiores níveis de conquista e desenvolvimento de literacias digitais, que refletem um maior grau de engajamento social, autonomia e expressão pessoal por parte dessa população. 
comunicação \& educação • Ano XVII • número 1 • jan/jun 2012

O levantamento e a análise dos dados sobre comportamento, usos e apropriações das TIC pela população idosa frequentadora do AcessaSP reveleram-se adequados e promissores para a abordagem netnográfica, na medida em que, consoante às proposições metodológicas de Kozinets ${ }^{21}$, evidenciou a existência de um público crescentemente conectado, adepto e ativo nas práticas comunicacionais digitais. Destacou-se, especialmente, a participação dos frequentadores de 60 anos ou mais na construção e uso de perfis em redes sociais e na produção de vídeos utilizando-se do YouTube, o que denota uma crescente e significativa apropriação das ferramentas da Web 2.0 por esse público. Foram relevantes, também, a participação em chats e salas de bate-papo e a criação ou atualização de blogs, microblogs e sites, entre outras ações nas quais se registram e se podem coletar e analisar a construção discursiva das representações sociais e geracionais, interações, simbologias e da própria participação e construção identitária no mundo virtual.

Esse trabalho constitui-se, assim, em um estudo inicial sobre as potencialidades do uso da netnografia na abordagem das comunidades virtuais, buscando abrir caminhos para futuras pesquisas não apenas do Núcleo de Pesquisa de Novas Tecnologias da Comunicação Aplicadas à Educação (NAP) "Escola do Futuro"/USP, mas, também, para outros pesquisadores envolvidos com as temáticas das literacias digitais emergentes na sociedade contemporânea e na inclusão social de grupos e populações econômica e/ou culturalmente marginalizados.

\section{REFERÊNCIAS BIBLIOGRÁFICAS}

CARDOSO, Gustavo. Os media na sociedade em rede. Lisboa: Fundação Calouste Gulbenkian, 2006.

CASTELLS, Manuel. A sociedade em rede. São Paulo: Paz e Terra, 1999.

COUNCIL OF AUSTRALIAN UNIVERSITY LIBRARIAN. Informations literacy standards [Padrões de literacias informacionais]. Canberra, 2001.

GARDNER, Howard. A nova ciência da mente: uma história da revolução cognitiva. São Paulo: Editora da Universidade de São Paulo, 2003.

GILSTER, Paul. Digital literacy [Literacia digital]. San Francisco, CA: John Willey \& Sons, 1997.

HARRIS, Benjamin. Communities as necessity in information literacy development: challenging the standards [Comunidades como necessidade do desenvolvimento de literacias informacionais: desafiando os padrões]. The Journal of Academic Librarianship, v. 34, n. 3, p. 248-255, maio 2008.

HARTLEY, John. Communication, Cultural and Media Studies: the key concepts. [Comunicação, Estudos Culturais e de Mídia: conceitos-chave]. Londres: Routledge, 2002.

HINE, Christine. Virtual Ethnography [Etnografia virtual]. London: Sage, 2000. 
Virtual Methods: Issues in Social Research on the Internet [Métodos virtuais: estudos em pesquisa social na internet]. Oxford: Berg, 2005.

JENKINS, Henry. Cultura da convergência. São Paulo: Aleph, 2008.

JOHNSTON, Bill; WEBBER, Sheila. As we may think: information literacy as a discipline for the information age [Como podemos pensar: literacias informacionais como uma disciplina para a Era da Informação]. Research Strategies, v. 20, n. 3, p. 108-121, 2006.

KERKHOVE, Derrick de. A pele da cultura: uma investigação sobre a nova realidade electrónica. Lisboa: Relógio D’água, 1997.

LLOYD, Annemaree. Information literacy landscapes: an emerging picture [Panorama das Literacias Informacionais: um quadro emergente]. Journal of Documentation, v. 62, n. 5, p. 570-583, 2006.

MARTÍN-BARBERO, Jesús. Cartografias culturales de la sensibilidad y la tecnicidad [Cartografias culturais da sensibilidade e da tecnicidade]. Apostila do curso ministrado no Programa de Pós-Graduação em Ciências da Comunicação na Escola de Comunicações e Artes (ECA) da Universidade de São Paulo, 2008.

PASSARELLI, Brasilina. Literacias emergentes nas redes sociais: estado da arte e pesquisa qualitativa no observatório da cultura digital. In: PASSARELLI, Brasilina; AZEVEDO, José (org.). Atores em rede: olhares luso-brasileiros. São Paulo: Editora Senac, 2010.

SINGH, Jagtar. Sense-making: information literacy for lifelong learning and information management [Sensemaking: literacias informacionais para o gerenciamento da informação e aprendizado ao longo da vida]. Journal of Library and Information Technology, v. 28, n. 2, p. 13-17, mar. 2008.

STREET, Brian. Social literacies: critical approaches to literacy in development, ethnography and education [Literacias Sociais: aproximações críticas das literacias em desenvolvimento, etnografia e educação]. London: Longman, 1995.

VASCONCELLOS, Marcelo Simão; ARAÚJO, Inesita Soares de. Usos da etnografia em mundos virtuais baseados em imagem. RECIIS: Revista Eletrônica de Comunicação, Informação e Inovação em Saúde, Rio de Janeiro, v. 5, n. 2, p. 75-85, 2011.

WARSCHAUER, Mark. Technology and social inclusion: rethinking the digital divide [Tecnologia e inclusão social: repensando a exclusão digital]. Massachusetts: MIT Press, 2003.

WEBBER, Sheila; JOHNSTON, Bill. Conceptions of information literacy: new perspectives and implications [Conceitos de literacia informacional: novas perspectivas e implicações]. Journal of Information Science, v. 26, n. 6. p. 381397, 2000. 
comunicação \& educação • Ano XVII • número 1 • jan/jun 2012

\section{Endereços eletrônicos}

BRAGA, Adriana. Usos e consumos de meios digitais entre participantes de weblogs: uma proposta metodológica. In: Anais do XVI Encontro da Compós, Curitiba, 2007. Disponível em: <http://compos.org.br/data/biblioteca_162. pdf $>$. Acesso em: 06 março 2011.

KOZINETS, Robert. The Field Behind the Screen: Using Netnography for Marketing Research in Online Communities [O campo atrás da tela: usando netnografia para pesquisas de Marketing em comunidades online]. Journal of Marketing Research, 39, February, p. 61-72, 2002. Disponível em: <http://www. nyu.edu/classes/bkg/methods/netnography.pdf $>$. Acesso em: 15 out. 2011.

KOZINETS, Robert; SALLA, Thiago Mio; TAVERNARI, Mariana. Literacias emergentes dos atores em rede: etnografia virtual com idosos no Programa de Inclusão Digital AcessaSP. Revista Prisma.com, n. 13, 2010. Disponível em: $<$ http://revistas.ua.pt/index.php/prismacom/article/view/789>. Acesso em: 10 maio 2011.

"School of the Future" - Research Laboratory/USP: action research and emerging literacies studies in WEB 2.0 environments [Escola do Futuro Laboratório de Pesquisa/USP: pesquisa-ação e estudos de literacias digital na Web 2.0]. The Journal of Community Informatics, v. 7, ns. 1 \& 2, 2010/2011. Disponível em: <http://ci-journal.net/index.php/ciej/article/view/646>. Acesso em: 14 out. 2011.

ROCHA, Paula Jung; MONTARDO, Sandra Portella. Netnografia: incursões metodológicas na cibercultura. e-COMPÓS: Revista da Associação Nacional dos Programas de Pós-Graduação em Comunicação, p. 13-22, dez. 2005. Disponível em: <http://www.compos.com.br/e-compos >. Acesso em: 22 nov. 2007. 